Recibido 05 de octubre, 2017 - Aceptado 05 de noviembre, 2017

\title{
Influencia de la actividad del zorro andino (Pseudalopes Culpaeus) sobre la crianza de ovinos, en una empresa ganadera del centro del Perú
}

\section{Influence of the activity of the andean fox (Pseudalopes Culpaeus) on the sheep raising, in a cattle company of the center of Peru}

\author{
Humberto Valenzuela, Maité Vaquerizo, Ignacio Ramírez, C. Pantoja, E. Rojas ${ }^{1}$
}

\section{RESUMEN}

El presente trabajo del tipo observacional, descriptivo, retrospectivo, se realizó en la SAIS Túpac Amaru, localizada en $-11.76^{\circ}$ latitud sur y longitud $-75.73^{\circ}$, sierra central del Perú. La altitud fluctúa entre 3600 a $4800 \mathrm{msnm}$, con temperatura que oscilan entre $-5^{\circ} \mathrm{C}$ a $18^{\circ} \mathrm{C}$. El objetivo fue evaluar e Investigar la influencia de la actividad del zorro andino sobre la crianza de ovinos, en una empresa ganadera del centro del Perú. Y los objetivos específicos: Analizar la tasa de mortalidad por causa de zorros en ovinos según clases. Analizar la tasa de prevalencia de ovinos enfermos a causa del zorro según clases de ganado. Formular estrategias de manejo y control ante la influencia de la actividad del zorro andino sobre la crianza de ovinos. Para lo cual se consideró toda la población de ovinos $(74,179)$ raza Junín.

El método utilizado fue el análisis de los registros de morbi y mortalidad de ovinos período 2011 - 2016, considerando clases de ganado. Los resultados muestran que los zorros han ocasionado una mortalidad de 23,938 ovinos durante el período de evaluación, la tasa media de mortalidad es de $5.12 \%$. Sin embargo esta tasa es creciente de un año a otro, siendo el incremento porcentual anual promedio de $2.12 \%$. Este hecho, constituye una realidad muy preocupante, debido a que merma el capital pecuario de la empresa. Las lesiones ocasionadas por los zorros, constituyen causas de morbilidad en el ganado ovino.

El número de casos promedio para un período anual es de $738 \pm 236.8$ ovinos enfermos por esta causa. Se concluye que la relación: Especies silvestres "zorro" - Especies domésticas "ovinos" y hombre, no guardan equilibrio, existiendo una actividad negativa de perjuicio y depredación de parte de los zorros (por un lado) y de constante labor de crianza y preocupación de parte del hombre (por otro lado). Se recomienda: Establecer inmediatamente un programa de control de Zorros, a través de la caza y reubicación definitiva, debiendo ser destinados a: Parques naturales de conservación, zoológicos u otros a fin de promover su conservación como especie silvestre y evitar la pérdida económica de la empresa.

Palabras claves: Zorro, Ovinos Junín.

1. Valenzuela H. Director de la Escuela Profesional de Ingeniería Zootécnica- Universidad Alas Peruanas. E-mail: h_valenzuela@uap.edu.pe 1. Baquerizo M. Coordinadora Académica de Escuela de Medicina Veterinaria-Universidad Alas Peruanas. E-mail: m_baquerizo@uap.edu. pe

1 Ramírez I. Decano de la Facultad de Ciencias Agropecuarias- Universidad Alas Peruanas. E-mail: i_ramirez@uap.edu.pe

2. Pantoja C. Docente de la Escuela de Ingeniería Zootécnica- Universidad Alas Peruanas. E-mail: pantoja4444@hotmail.com

2. SAIS Túpac Amaru - Junín - Perú 


\section{ABSTRACT}

The present work of the observational, descriptive, retrospective type was carried out in SAIS Túpac Amaru, located at $-11.76^{\circ}$ south latitude and longitude $-75.73^{\circ}$, central highlands of Peru. The altitude fluctuates between 3600 to $4800 \mathrm{msnm}$, with temperature that oscillate between $-5^{\circ} \mathrm{C}$ to $18^{\circ} \mathrm{C}$. The objective was to evaluate and investigate the influence of the activity of the Andean fox on the breeding of sheep, in a livestock company in central Peru. And the specific objectives: To analyze the mortality rate due to foxes in sheep according to classes. Analyze the prevalence rate of sick sheep due to the fox according to livestock classes. Formulate strategies of management and control before the influence of the activity of the Andean fox on the breeding of sheep. For which the entire population of sheep $(74,179)$ Junín race was considered.

The method used was the analysis of the records of morbi and mortality of sheep period 2011 - 2016, considering livestock classes. The results show that foxes have caused a mortality of 23,938 ovines during the evaluation period, the average mortality rate is $5.12 \%$. However, this rate is increasing from one year to the next, with an average annual percentage increase of $2.12 \%$. This fact is a very worrying reality, because it reduces the livestock capital of the company and above all leads to economic losses. Injuries caused by foxes are causes of morbidity in sheep.

The average number of cases for an annual period is $738 \pm 236.8$ diseased sheep due to this cause. It is concluded that the relationship: Wild species "fox" - Domestic species "sheep" and man, do not keep balance, there being a negative activity of prejudice and depredation on the part of foxes (on the one hand) and constant breeding and concern on the part of man (on the other hand). It is recommended: Immediately establish a Fox control program, through hunting and definitive relocation, should be allocated to: Natural conservation parks, zoos or others in order to promote their conservation as a wild species and avoid economic loss of company.

Keywords: Fox, Junin Sheep

\section{INTRODUCCIÓN}

La crianza de ovinos y la necesidad de llegar a una producción de mejor y mayor calidad cada vez se va convirtiendo en una necesidad imperiosa entre los criadores y los centros experimentales en el Perú.

Hasta la actualidad según el Último Censo Agropecuario realizado por el INEI el 2012 contamos con una población ovina a nivel nacional de 9'523,198 cabezas de ganado ovino, de las cuales la mayor cantidad de ovinos se encuentra concentrada en la sierra con un porcentaje de $(94,2 \%)$. Siendo el $(80,5 \%)$ ovino criollo; el $(11,3 \%)$ Corriedale; el $(2,6 \%)$ Hapshire Down; el

\section{(0,9\%) Black Belly y el (4,1\%) Otros.}

Como podemos apreciar hay un porcentaje mayor de ovinos criollos, no podemos negar la rusticidad de este animal que se adaptación facilidad a los diferentes climas del Perú.

La crianza presenta dos niveles tecnológicos de producción; el nivel alto, que involucra al 25\% de la población ovina, en propiedad de empresas asociativas campesinas y medianos productores.

Sin embargo, existen algunos factores que atenta contra estos esfuerzos, sobre todo, el que se relaciona con la fuerte pérdida de animales, producto de la depredación de animales por el zorro. 
Estos carnívoros han mantenido a lo largo de la historia una relación mayoritariamente conflictiva con el hombre. En gran medida ello responde a los hábitos predatorios de estos animales, a una dieta de alto nivel proteico y áreas de acción generalmente extensas que los exponen a una competencia recurrente con el ser humano, quien en cierta forma tiene las mismas demandas (Treves y Karanth, 2003).

Tal conflicto ancestral, si bien es más evidente en los carnívoros de mayor tamaño, también se manifiesta en especies de tamaño mediano y pequeño.

Los zorros presentan una dieta alimenticia variada según estudios realizados en argentina y citados por Manero (2001) y en Chile, por Jiménez y Novaro (2004), en la que se incluyen liebre europea, ovinos, roedores, aves y otros animales.

El zorro generalmente ataca a animales pequeños y a crías, particularmente a corderos y cabritos. Las mordidas características se limitan a la zona del cuello y costillar y muestran predilección por las vísceras.

En el caso del zorro gris o chilla son animales omnívoros y su dieta está compuesta de mamíferos, artrópodos, aves, reptiles, frutas y carroñas y cuando atacan animales vivos, por lo general, son mamíferos de pequeño tamaño (González del Solar y Rau, 2004).

Por todo lo expuesto, en el presente estudio se planteó los siguientes objetivos:

\section{Objetivo General:}

- Evaluar e Investigar la influencia de la actividad del zorro andino sobre la crianza de ovinos, en una empresa ganadera del centro del Perú.

\section{Objetivos Específicos:}

- Analizar la tasa de mortalidad por causa de zorros en ovinos según clases.
- Analizar la tasa de prevalencia de ovinos enfermos a causa del zorro según clases de ganado. - Formular estrategias de manejo y control ante la influencia de la actividad del zorro andino sobre la crianza de ovinos.

\section{Hipótesis:}

Hi: La morbilidad a causa de zorros tiene alta prevalencia en ovinos de raza Junín y como consecuencia, genera altas pérdidas económicas.

Ho: La morbilidad a causa de zorros tiene baja prevalencia en ovinos de raza Junín y por tanto, no genera pérdidas económicas.

\section{MATERIALES Y MÉTODOS}

\section{Localización}

El presente trabajo de investigación, se realizó en la SAIS Túpac Amaru, localizada en $-11.76^{\circ}$ latitud sur y longitud $-75.73^{\circ}$, jurisdicción de Pachacayo, distrito de Canchayllo, provincia de Jauja, departamento de Junín, sierra central del Perú.

Los campos de pastoreo se hallan entre $3700 \mathrm{y}$ $4500 \mathrm{msnm}$ de altitud con temperaturas que oscilan entre $-5^{\circ} \mathrm{C}$ a $18^{\circ} \mathrm{C}$ y exhiben un relieve topográfico relativamente suave y variando con pendientes de $2 \%$ a $50 \%$ y, en un menor grado, el cuadro topográfico se completa con la presencia de montañas, áreas nivales, escarpes y riveras lacustres.

La pluviosidad promedio anual observada es de $650 \mathrm{~mm}$ entre 4100 y $4800 \mathrm{~m}$ de altitud, de 800 $\mathrm{mm}$ entre 4100 y $4800 \mathrm{~m}$ de altitud y de $900 \mathrm{~mm}$ sobre $4800 \mathrm{msnm}$.

El ambiente de la sierra alta y puna se caracteriza por, una acentuada sequía, raleza del oxígeno en el aire, alta luminosidad y radiación ultravioleta. 


\section{De los animales}

Los animales del presente estudio corresponden a la raza Junín, cuyas características fenotípicas, se describen a continuación: Los carneros exhiben una conformación equilibrada, son de gran tamaño y fuertes; tienen pecho amplio y cuerpo de notable profundidad y largo.

La estampa del carnero Junín destaca una cabeza fuerte sin cuernos, cara amplia y descubierta de lanas. Las extremidades son largas y fuertes. Los carneros adultos pesan en promedio $75 \mathrm{~kg}$.

Lo que es verdaderamente sobresaliente para ovinos pastoreados extensivamente y a campo abierto, en la sierra alta y puna.

La oveja se destaca por una notoria cualidad maternal, por su corpulencia, fortaleza en el tren posterior y una buena capacidad lechera, las ovejas adultas tienen un peso vivo promedio de 46 kg. y los corderos llegan a pesar $23 \mathrm{~kg}$. al destete y $36 \mathrm{~kg}$. a los 8 meses de edad. Villarroel y Gamarra (1978).

\section{Del régimen de alimentación y sistema de crianza}

La alimentación de los ovinos del presente estudio, es sobre praderas naturales en el que existe una marcada predominancia de gramíneas y especies perennes tales como: Chilligua (Festuca dolichophylla), Crespillo (Calamagrostis vicunarum), Sillu sillu (Alchemilla pinnata), Dissattelium minimum, Stipa brachyphylla, Agrostis breviculmis y Muhlenbergia fastigiata. La disponibilidad de agua es ad libitum.

El sistema de crianza es bajo el sistema extensivo, al pastoreo sobre 216,000 hás aproximadamente de praderas de pastos naturales.

\section{Metodología}

\section{Población y muestra}

En el presente estudio, se consideró la totalidad de la población de ovinos con que cuenta la empresa $(74,179)$.

\section{Obtención de datos}

Se procedió al análisis de los registros de morbi y mortalidad de ovinos de cuatro años (2011 2016), considerando clase de ganado.

Todos los datos recopilados, fueron tabulados y luego analizados mediante estadística descriptiva: media, varianza, desviación estándar, coeficiente de variación. Así mismo se determinó la tasa de morbilidad y prevalencia de periodo.

\section{RESULTADOS}

\section{De la mortalidad de ovinos según clases}

La incidencia del zorro andino varía de acuerdo a la clase de ganado, por lo que el reporte indica a los corderos como la clase más afectada con un total de 11858 casos, seguido de las borregas que han presentado 4236 casos para las borreguillas un total de 3776 muertes, los carnerillos presentan 1304 casos y para la clase de ganado que menos afectada se ve son los capones con un total de 297 casos.

Por lo tanto la empresa ganadera S.A.I.S TUPAC AMARU, en el período de 6 años (2011-2016) ha registrado un total de 23,938 muertes por ataques de zorro lo que afecta directamente en la cría del ganado ovino, traduciendo esto en pérdidas económicas, disminuyendo la rentabilidad de la empresa. Tabla 1. 
Tabla No 1 . Número de animales muertos por zorro, según clase

\begin{tabular}{|c|c|c|c|c|c|c|c|c|}
\hline \multirow{2}{*}{ AÑo } & \multicolumn{7}{|c|}{ CLASE } & \multirow{2}{*}{ TOTAL } \\
\hline & BORREGAS & CORDEROS & BORREGUIUAS & CAPONCILOS & CARNERILOS & CARNEROS & CAPONES & \\
\hline 2011 & 672 & 2100 & 514 & 266 & 171 & 43 & 80 & 3846 \\
\hline 2012 & 698 & 2002 & 550 & 294 & 207 & 21 & 34 & 3806 \\
\hline 2013 & 507 & 1711 & 629 & 366 & 142 & 21 & 58 & 3434 \\
\hline 2014 & 793 & 1843 & 598 & 422 & 234 & 54 & 44 & 3968 \\
\hline 2015 & 911 & 2549 & 803 & 414 & 320 & 118 & 47 & 5162 \\
\hline 2016 & 655 & 1653 & 682 & 368 & 230 & 100 & 34 & 3722 \\
\hline TOTAL & 4236 & 11858 & 3776 & 2130 & 1304 & 357 & 297 & 23938 \\
\hline MEDIA & 706,00 & 1976,33 & 629,33 & 355,00 & 217,33 & 99,50 & 49,50 & 3998,67 \\
\hline DS & 136,39 & 327,60 & 103,45 & 63,09 & 61,49 & 40,82 & 17,43 & 601,60 \\
\hline $\mathrm{CV}$ & 19,32 & 16,58 & 16,44 & 17,77 & 28,29 & 68,60 & 35,22 & 15,08 \\
\hline
\end{tabular}

Al evaluar la tabla 2, se observa que la tasa media de mortalidad es de $5.12 \%$. Sin embargo esta tasa es creciente de un año a otro, siendo el incremento porcentual anual promedio de $2.12 \%$. Este hecho, constituye una realidad muy preocupante, debido a que merma el capital pecuario de la empresa y sobre todo conlleva a pérdidas económicas.

Grafico No1. Número de ovinos muertos, período 2011 a 2016.

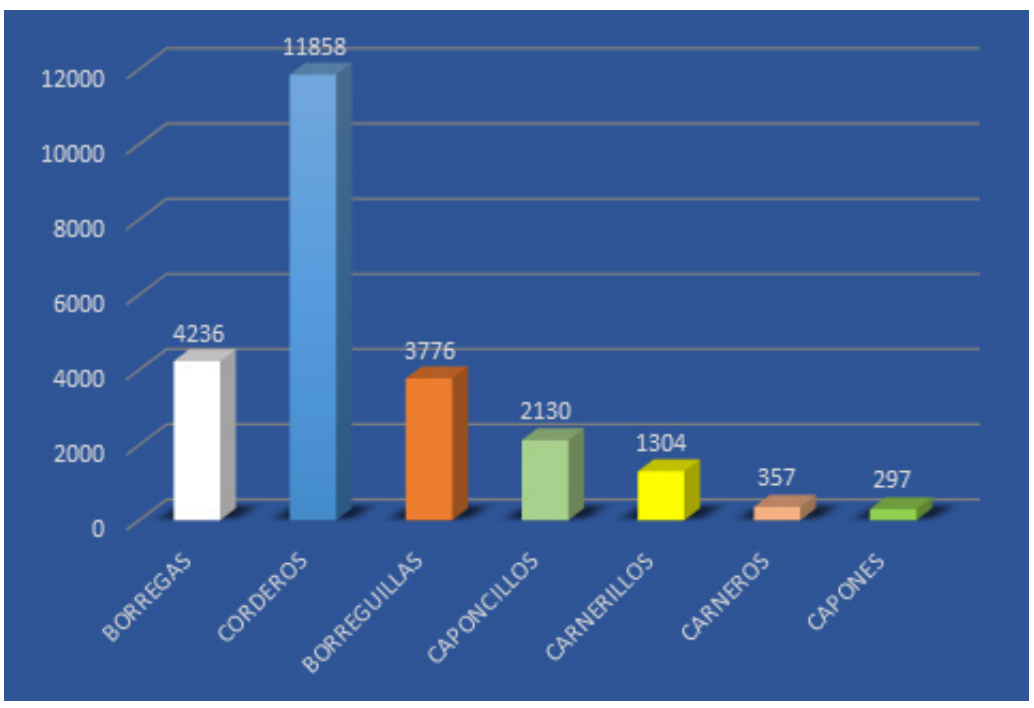

Tabla No2. Tasa de mortalidad (\%) en ovinos de raza Junín, según clase

\begin{tabular}{|c|c|c|c|c|c|c|c|c|}
\hline & \multicolumn{7}{|c|}{ CLASE } & \multirow{2}{*}{} \\
\cline { 2 - 8 } ANNO & BORREGAS & CORDEROS & BORREGULLASCAPONGLLOS & CARNERLLOS & CARNEROS & CAPONES & TOTAL \\
\hline 2012 & 1,93 & 7,67 & 5,61 & 7,87 & 5,87 & 0,57 & 6,56 & 4,56 \\
\hline 2013 & 1,39 & 6,52 & 7,06 & 10,37 & 4,14 & 0,53 & 7,76 & 4,12 \\
\hline 2014 & 2,19 & 7,44 & 6,36 & 11,20 & 6,29 & 1,41 & 14,15 & 4,84 \\
\hline 2015 & 2,62 & 11,96 & 9,94 & 19,14 & 10,72 & 2,72 & 8,06 & 6,96 \\
\hline
\end{tabular}




\section{De la morbilidad de ovinos a causa de zorros, según clases}

Las lesiones ocasionadas por los zorros, constituyen causas de morbilidad en el ganado ovino. En la tabla 3, podemos apreciar que el número de casos promedio para un período anual es de $738 \pm 236.8$ ovinos enfermos por esta causa.

Este hecho, también genera pérdidas económicas a la empresa, debido a que genera gastos de tratamientos y cuidados especiales que podrían terminar en muerte.

Tabla No3. Número de animales enfermos y/o accidentados por zorro, según clase

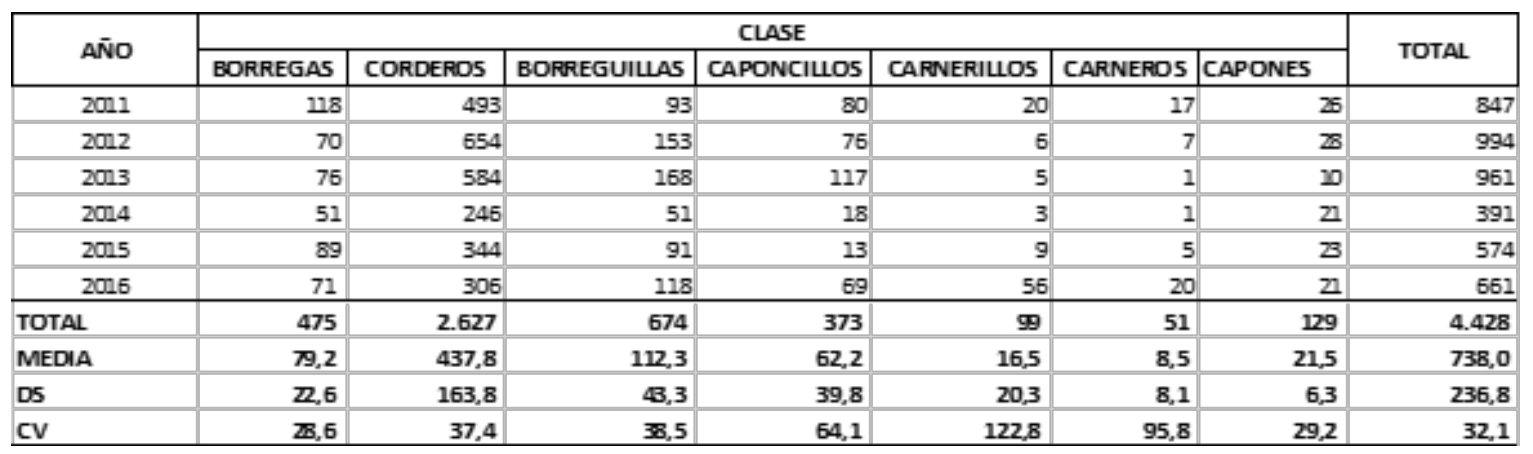

$\mathrm{Al}$ analizar la prevalencia de período (\%), observamos que los corderos y los capones, son las clases de ganado que más tasa de prevalencia muestran (corderos: min 0.99 - máx 2.51; capones: min 1.34 máx 6.75). Estos valores pueden explicados por la alta presencia de zorros en los lugares de pastoreo durante la época de nacimientos, atraídos por los residuos placentarios localizados.

Tabla No4. Prevalencia de período (\%) de ovinos enfermos a causa de accidentes de zorro, según clase

\begin{tabular}{|c|c|c|c|c|c|c|c|c|}
\hline \multirow[b]{2}{*}{ AÑO } & \multicolumn{7}{|c|}{ CLASE } & \multirow[b]{2}{*}{ TOTAL } \\
\hline & BORREGAS & CORDEROS & BORREGUILLAS & CAPONCILLOS & CARNERILLOS & CARNEROS & CAPONES & \\
\hline 2012 & 0,19 & 2,51 & 1,56 & 2,03 & 0,17 & 0,19 & 5,41 & 1,19 \\
\hline 2013 & 0,21 & 2,22 & 1,88 & 3,32 & 0,15 & 0,03 & 1,34 & 1,15 \\
\hline 2014 & 0,14 & 0,99 & 0,54 & 0,48 & 0,08 & 0,03 & 6,75 & 0,48 \\
\hline 2015 & 0,26 & 1,61 & 1,13 & 0,60 & 0,30 & 0,12 & 3,95 & 0,77 \\
\hline
\end{tabular}

De todos los resultados obtenidos en el presente estudio, podemos deducir que la relación: Especies silvestres "zorro" - Especies domésticas "ovinos" y hombre, NO GUARDAN EQUILIBRIO, existiendo una actividad negativa de perjuicio y depredación de parte de los zorros (por un lado) y de constante labor de crianza y preocupación de parte del hombre (por otro lado). 


\section{Gráfico No2. Tasa de prevalencia de período de ovinos enfermos a causa de accidentes de zorro, según clase}

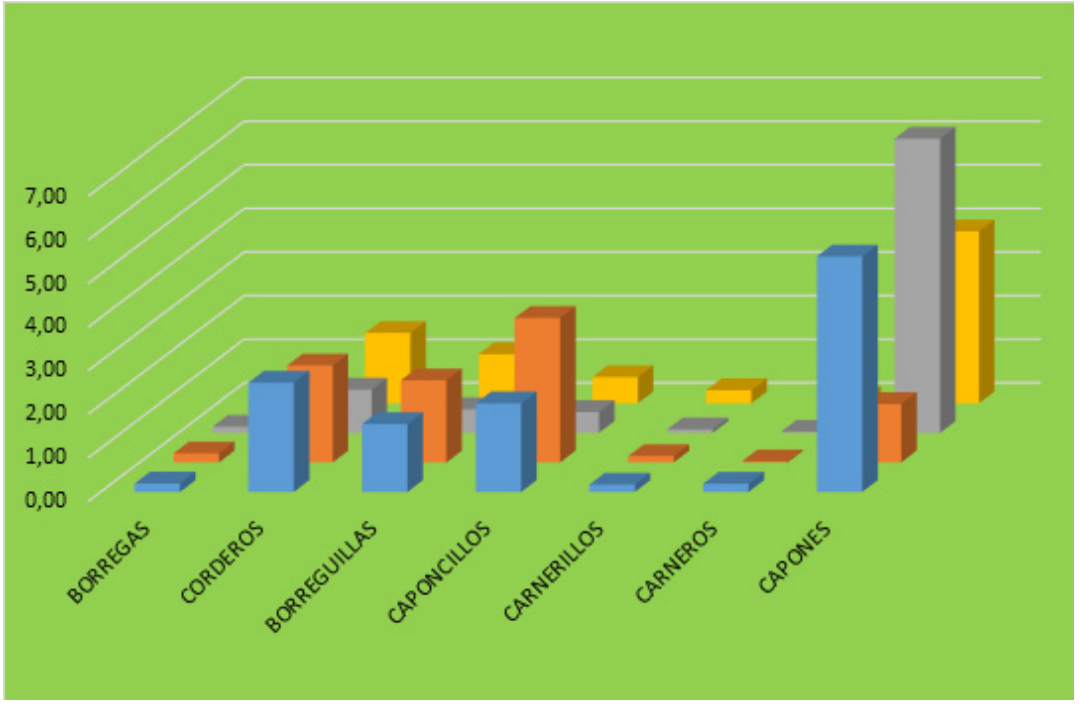

Al respecto muchos autores abordan la actividad del zorro del siguiente modo: Crespo 1971, reporta que la dieta del Pseudalopex gymocercus en la pampa argentina está constituido de $25 \%$ por alimento de origen vegetal y $75 \%$ de origen animal.

Falero (1988) reporta que en la dieta del Pseudalopex culpaeus, en zonas de crianza de ganado caprino nunca supero el $5 \%$ en las Lomas de Lachay, que comparado con el presente estudio supera dicha tasa.

\section{CONCLUSIONES}

\section{De acuerdo a los resultados del presente estu-} dio, se concluye:

-No existe equilibrio en relación a la actividad del zorro andino y la actividad productiva de la SAIS Túpac Amaru.

-Analizando la población estimada de Zorros en la zona, respecto a la población existente de ganado ovino, ésta es minoritaria ó mínima (no se tiene información cuantificada). Sin embargo ocasiona pérdidas cuantiosas de gran magnitud: 28,366 ovinos afectados por zorros en un período de 6 años.
La relación hombre: especie silvestre, en estas condiciones observadas, genera un efecto de rechazo y se busca la eliminación o extinción de esta especie silvestre, hecho que generaría desequilibrio ecológico y/o la pérdida definitiva.

\section{RECOMENDACIONES}

-Establecer inmediatamente un programa de control de Zorros, a través de la caza y reubicación definitiva, debiendo ser destinados a: Parques naturales de conservación, zoológicos $\mathrm{u}$ otros a fin de promover su conservación como especie silvestre y evitar la pérdida económica de la empresa.

-Establecer un procedimiento de eliminación de residuos placentarios en el campo de pastoreo de los ovinos, así como de los corderos muertos en campo por diferentes causas.

-Considerar el establecimiento de un núcleo de perros, destinados a ahuyentar a los zorros de los campos de pastoreo de ovinos, especialmente durante la época de parición. 


\section{Agradecimiento}

Expresamos nuestro agradecimiento a la empresa SAIS Túpac Amaru y sus trabajadores por brindarnos las facilidades durante el presente estudio. En especial al Ing. Fredy Rojas, Ing. Renato Huayre. Así mismo a los alumnos: García L., Moscoso K., Aquiño J., Hurtado C, quienes participaron durante el proceso de la presente investigación.

\section{BIBLIOGRAFIA}

Borrelli, P, G. Oliva, M. Williams, L. González, P. Rial y L. Montes. 1997. Sistema Regional de Soporte de Decisiones - Santa Cruz y Tierra del Fuego. PRODESER (INTA - GTZ). E.E.A. Santa Cruz, Santa Cruz, Argentina. 136 pp.

Howard, W. A. 1976. A philosophy of vertebrate pest control. Proceedings of the 7th Vertebrate Pest Conference.

INSTITUTO NACIONAL DE ESTADISTICA E INFORMATICA (INEI) 2012. Avances del IV Censo Nacional Agropecuario 2012. Lima - Perú.

Lycalopex culpaeus andinus. https://es.wikipedia.org/wiki/ Lycalopex_culpaeus_andinus

Manero, A. 2001 La acción del zorro colorado en la producción ovina. Cap. 12. pp 243-252. En: Ganadería Sustentable en la Patagonia Austral. Borrelli, P. y G. Oliva Ed. INTA Reg. Pat. Sur. 269 pp.

Manual de manejo ovino Instituto de Desarrollo Agropecuario - Instituto de Investigaciones Agropecuarias. Editoras: Verónica González M. / Marilyn Tapia M. BOLETÍN INIA / Nº 03 INDAP, Santiago 2017.

Manual de ovinos., Las buenas practicas Abril 1ra. Edición 2013, Dirección General de Competitividad Agraria. Ing. Rosario Isabel Díaz Ramírez - MVZ.SC. Dr. Hugo Vilcanqui Mamani.

Ministerio del Ambiente y Desarrollo Sustentable. Dirección de Fauna Silvestre y Conservación de la Biodiversidad, Uso sustentable y conservación de zorros en Argentina Proyecto zorros.

Presencia de enfermedades parasitarias e infecciosas (Leptospirosis, distemper y brucelosis) en zorros andinos (Lycalopex culpaeus) que habitan en los páramos de la Hacienda Antisanilla (Pintag-Ecuador). Nicole Veintimilla Andrés Ortega, MVZ., Director de Tesis.
Reproduccion de zorro. http://www.zorropedia.com/reproduccion-zorro/

Zorro Andino - Pseudalopex culpaeus. http://animalesyplantasdeperu.blogspot.pe/2007/04/el-zorro-andino.html. 